\title{
Efficacy Study With Pre-Exposure Prophylaxis
}

National Cancer Institute

\section{Source}

National Cancer Institute. Efficacy Study With Pre-Exposure Prophylaxis. NCI Thesaurus. Code C158356.

A study that assesses the efficacy of prophylactic treatment (including radioprotectors) given before exposure to an agent(s). 